\title{
Bilateral Tubal Molar and Embryonic Pregnancy
}

\author{
Hyacinthe Zamane ${ }^{1,2^{*}}$, Aimé Sosthène Ouedraogo 2,3, Yobi Alexis Sawadogo ${ }^{1,2}$, \\ Barnabé Yameogo1, Yacinthe Sidbéwenné Kabore ${ }^{4}$, Paul Dantola Kain 1,2, \\ Sibraogo Kiemtore ${ }^{1,2}$, Adama Ouattara1,2, Blandine Bonane Thieba ${ }^{1,2}$
}

\footnotetext{
${ }^{1}$ Department of Obstetrics and Gynecology, Yalgado Ouedraogo Teaching Hospital, Ouagadougou, Burkina Faso

${ }^{2}$ Health Sciences Research Training Unit, University of Ouagadougou, Ouagadougou, Burkina Faso

${ }^{3}$ Anatomo Pathologic Unit, Yalgado Ouedraogo Teaching Hospital, Ouagadougou, Burkina Faso

${ }^{4}$ Unity of Obstetrics and Gynecology, Regional Hospital of Tenkodogo, Tenkodogo, Burkina Faso

Email: *zamanehyacinthe@gmail.com, zamanehyacinthe@yahoo.fr
}

How to cite this paper: Zamane, H., Ouedraogo, A.S., Sawadogo, Y.A., Yameogo, B., Kabore, Y.S., Kain, P.D., Kiemtore, S., Ouattara, A. and Thieba, B.B. (2018) Bilateral Tubal Molar and Embryonic Pregnancy. Open Journal of Obstetrics and Gynecology, 8, 230-235.

https://doi.org/10.4236/ojog.2018.83024

Received: January 28, 2018

Accepted: March 13, 2018

Published: March 16, 2018

Copyright $(0) 2018$ by authors and Scientific Research Publishing Inc. This work is licensed under the Creative Commons Attribution International License (CC BY 4.0).

http://creativecommons.org/licenses/by/4.0/

\begin{abstract}
Aim: To report a case of bilateral tubal molar and embryonic pregnancy diagnosed and treated at the Yalgado Ouedraogo Teaching Hospital in Ouagadougou, Burkina Faso. Observation: It was an 18-year-old patient, G2P0 with no known pathological history. She was received in obstetric emergencies for suspicion of ectopic pregnancy in a two-month amenorrhea context. At the admission, the physical examination had found a sensitivity of the hypogastric region with a cry of the umbilicus and the bleeding. At pelvic ultrasound, the uterus was empty with a medium-abundance pelvic effusion and a left extra uterine mass. The initial rate of beta HCG was $5700 \mathrm{ml}$ IU per ml. A framed laparotomy was carried out. The operative exploration has revealed a right tubal pregnancy cracked and a left unbroken tubal pregnancy. The diagnosis of a bilateral ectopic pregnancy was retained. A right salpingectomy and a left tubal caesarean section were carried out. Histological analysis confirmed the diagnosis of a left embryonic ectopic pregnancy associated with a right molar ectopic pregnancy. Conclusion: The systematic use of histological analysis for any extra uterine pregnancy treatment is required to not ignore an associated molar pregnancy.
\end{abstract}

\section{Keywords}

Ectopic, Bilateral, Molar, Embryonic, Pregnancy

\section{Background}

Molar pregnancy is rare and its frequency varies from a geographic area to 
another. The risk factors for its occurrence are multiple and entangled [1]. Usually intrauterine, the molar pregnancy can be more rarely extra uterine [2] [3] [4] [5] or exceptionally heterotopic [6]. Vanita [7] reported a case of ectopic mole associated with a normal intrauterine pregnancy. Bilateral ectopic pregnancy is rare [8] [9]. The subsequent prognosis of fertility is uncertain with a risk of infertility or recurrence of ectopic pregnancy. This is a real concern in a context where access to medically assisted procreation is limited. Ectopic molar pregnancy simultaneously associated with a contralateral ectopic embryonic pregnancy is a more exceptional entity. Besides the fertility problem, the risk of persistent trophoblastic disease is to be feared. We report a case diagnosed and treated at the Yalgado Ouedraogo Teaching Hospital in Ouagadougou.

\section{Observation}

It was an 18-year-old patient with no gainful activity, G2P0. She had already made a spontaneous miscarriage and had no other known pathological antecedents. She was received in obstetric emergencies for acute pelvic pain and bleeding in a two-month amenorrhea context. At the admission the general condition was relatively kept. The physical examination found sensitivity of the hypogastric region and the bleeding. Pelvic ultrasound found an empty uterus, a medium-abundance pelvic effusion, with a 3-cm left extra uterine mass (Figure 1). The initial rate of beta HCG was $5700 \mathrm{ml}$ IU per ml. An ectopic pregnancy was suspected. The patient had received an emergency laparotomy. Post-operative exploration after aspiration of a medium abundance hem peritoneum highlighted a cracked right ectopic pregnancy. The left proboscis was the seat of an unbroken pregnancy of $3 \mathrm{~cm}$ of large axis (Figure 2).The diagnosis of a bilateral ectopic pregnancy was retained. A right salpingectomy and a left tubal caesarean section were performed. The anatomy pathology examination of the surgical parts showed an embryo on the left hand piece and numerous vesicles of varying size reaching 3 to $5 \mathrm{~mm}$ in diameter on the right salpingectomy piece.

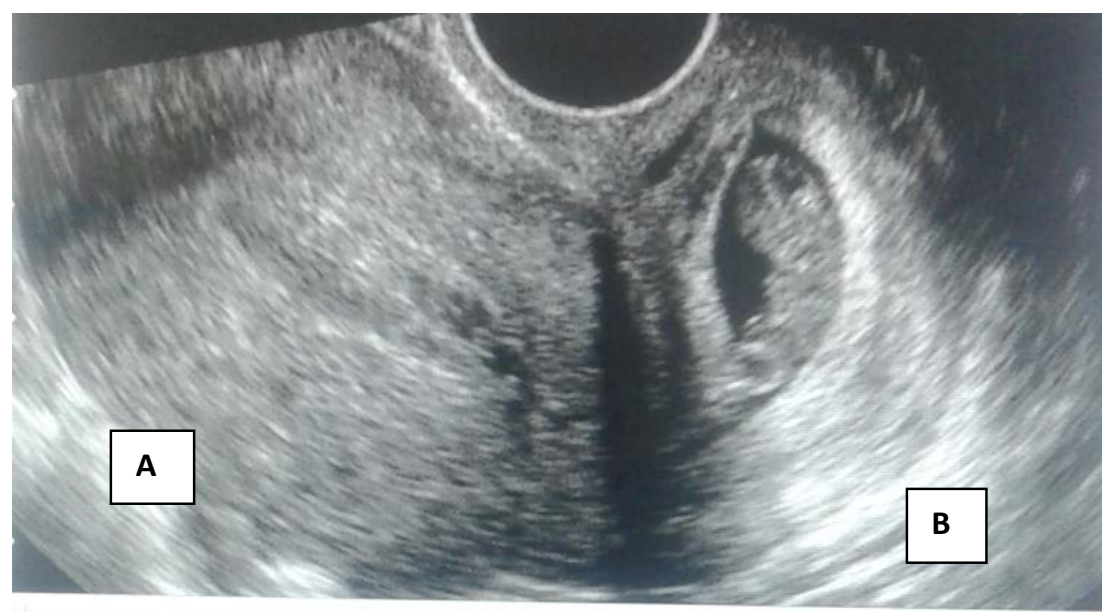

Figure 1. Ultrasound aspect. A: Empty uterus with a thickened endometrium; B: Left extra uterine mass. 


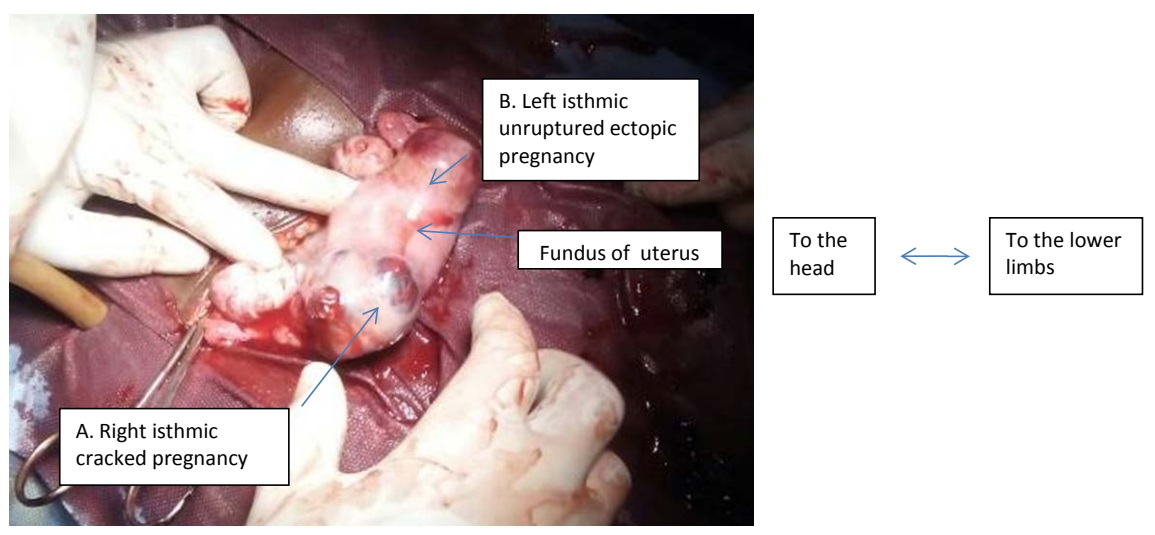

Figure 2. Per operative view of bilateral ectopic pregnancy. A: Right isthmic cracked pregnancy (site of molar pregnancy); B: Left isthmic unruptured ectopic pregnancy.

These vesicles were translucent and clustered in grapes. Histologically, these vesicles were hydro spades, avascular irregularly contoured with intussusceptions of the hyperplastic trophoblastic coating (Figure 3 ). In the end histology confirmed the diagnosis of right ectopic molar pregnancy associated with an embryonic left tubal pregnancy. The postoperative evolution was favorable. The patient was monitored by a regular dose of $\beta$-HCG under combined hormonal contraception. A convert of the $\beta$-HCG rate was observed after seven weeks.

\section{Comments}

Ectopic molar pregnancy is rare [2]. Its impact could be underestimated due to the lack of systematic practice of histological analysis on ectopic pregnancy surgical parts in some areas [11]. The ectopic mole in a context of bilateral ectopic pregnancy is exceptional. Pre-operative diagnosis of extra uterine molar pregnancy is difficult and exceptional [2] [10]. It is usually late especially in limited resource countries [11] and is frequently done at the stage of rupture [12] [13].

Clinically the ectopic mole mimics ectopic embryonic pregnancy which makes its pre-operative diagnosis difficult [14]. In addition to the delay in diagnosis, the frequency of rupture of the molar extra-uterine pregnancy would be related to a greater invasive potential of the mole compared to the embryonic pregnancy [2]. Due to a deficiency of vascularization in the ectopic position [2], the rate of Beta HCG is lower in the case of extra molar pregnancy than intra-uterine mole [14]. Therefore its dosage does not allow steering towards the molar nature of the ectopic pregnancy. Also the ultrasound diagnosis of mole is difficult to assert in early pregnancy [15] [16] [17] which can lead to the use of MRI [2]. In our patient, only the embryonic ectopic pregnancy had been visualized on the ultrasound.

Histology is a great contribution to diagnosis. The management of extra-uterine pregnancy is variable [9] which can be medical, surgical conservative or radical according to multiple criteria [18]. Evolution is generally favorable [2] [19]. However, the conservative treatment exposes the risk of recurrence of extra-uterine pregnancy and the persistence of trophoblastic disease for the molar 


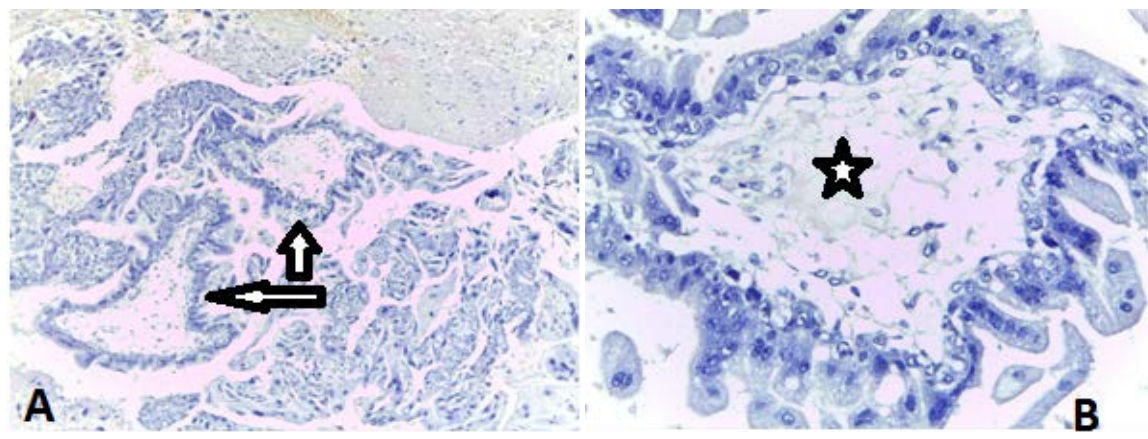

Figure 3. Staining Haematein eosin (HE). A) G 100. Trophoblastic proliferation of variable-sized vesicles (arrows), irregularly contoured and avascular hydro sting axis with hyperplasic trophoblastic coating; B) G 400. High magnification showing a hydro villus-axis (star) with trophoblastic coating invaginas.

component [18] [20]. Also, it would not significantly improve fertility [18] [21]. In view of the young age of our patient (18 years) and her low economic power not allowing him an easy recourse to medically assisted procreation, a salpingectomy carrying the right proboscis, seat of the molar pregnancy and a left tubal caesarean were realized. However, the subsequent prognosis of fertility in such a case remains reserved and the patient has received counselling in this direction.

\section{Conclusion}

The molar and embryonic bilateral pregnancy is exceptional. The systematic use of histological analysis for any extra uterine pregnancy treatment is required to avoid missing the diagnosis of an ectopic mole. The desire to preserve subsequent fertility and the risk of persistent trophoblastic disease are to be weighed when deciding the therapeutic option.

\section{References}

[1] Dreyfus, M., Tissier, I. and Philippe, R. (2000) Les maladies trophoblastiques gestationnelles. Classifications, épidémiologie et bades génétiques. Journal de Gynécologie Obstétrique et Biologie de la Reproduction, 29, 125-130.

[2] Yamada, Y., Ohira, S., Yamazaki, T. and Shiozawa, T. (2016) Ectopic Molar Pregnancy: Diagnostic Efficacy of Magnetic Resonance Imaging and Review of the Literature. Case Reports in Obstetrics and Gynecology, 2016, Article ID: 7618631. https://doi.org/10.1155/2016/7618631

[3] Gillespie, A.M., Lidbury, E.A., Tidy, J.A. and Hancock, B.W. (2004) The Clinical Presentation, Treatment, and Outcome of Patients Diagnosed with Possible Ectopic Molar Gestation. International Journal of Gynecological Cancer, 14, 366-369. https://doi.org/10.1111/j.1048-891X.2004.014223.x

[4] Burton, J.L., Lidbury, E.A., Gillespie, A.M., Tidy, J.A., Smith, O., Lawry, J., et al. (2001) Over-Diagnosis of Hydatidiform Mole in Early Tubal Ectopic Pregnancy. Histopathology, 38, 320-321. https://doi.org/10.1046/j.1365-2559.2001.01151.x

[5] Gavez, C.R., Fernandez, V.C., De Los Reves, J.M., Jaén, M.M. and Teruel, R.G. (2004) Primary Tubal Choriocarcinoma. International Journal of Gynecological 
Cancer, 14, 1040-1044. https://doi.org/10.1111/j.1048-891X.2004.014550.x

[6] Leung, F., Terzibachian, J.J., Chung Fat, B., Lassabe, C., Knoepffler, F., Maillet, R., et al. (2009) Heterotopic Ovarian Hydatidiform Mole. A Case Report. Gynecologie, Obstetrique \& Fertilite, 37, 749-751. https://doi.org/10.1016/j.gyobfe.2009.04.025

[7] Vanita, V. (2014) A Very Rare Case of Heterotopic Pregnancy in Natural Conception with Ectopic Pregnancy as Partial Mole. The Journal of Obstetrics and Gynecology of India, 64, 433-435. https://doi.org/10.1007/s13224-013-0426-y

[8] Jamilian, M. (2014) Bilateral Tubal Ectopic Pregnancy Following Intra Uterine Insemination (IUI): A Case Report. Iranian Journal of Reproductive Medicine, 12, 155-158.

[9] Jena, S.K., Singh, S., Nayak, M., Das, L. and Senapati, S. (2016) Bilateral Simultaneous Tubal Ectopic Pregnancy: A Case Report, Review of Literature and a Proposed Management Algorithm. Journal of Clinical and Diagnostic Research, 10, QD01-QD03. https://doi.org/10.7860/JCDR/2016/16521.7416

[10] Asseryanis, E., Schurz, B., Eppel, W., Wenzl, R., Vavra, N. and Husslein, P. (1993) Detection of an Atypical Invasive Mole in An Ectopic Pregnancy by Transvaginal Color-Flow Doppler. American Journal of Obstetrics and Gynecology, 169, 1656. https://doi.org/10.1016/0002-9378(93)90463-S

[11] Bambara, M., Diallo, A.A. and Lompo Goumbri, O. (2016) Molar Ectopic Pregnancy: A Case Study from the Maternity Unit of the Bobo-Dioulasso Teaching Hospital. Open Journal of Obstetrics and Gynecology, 6, 846-850.

https://doi.org/10.4236/ojog.2016.613102

[12] Berlingieri, P., Bogdanskiene, G. and Grudzinskas, J.G. (2007) Rupture of Tubal Pregnancy in the Vilnius Population. European Journal of ObstetricsGynecology \& Reproductive Biology, 131, 85-88. https://doi.org/10.1016/j.ejogrb.2006.03.004

[13] Frates, M.C., Doubilet, P.M., Peters, H.E. and Benson, C.B. (2014) Adnexal Sonographic Findings in Ectopic Pregnancy and Their Correlation with Tubal Rupture and Human Chorionic Gonadotropin Levels. Journal of Ultrasound in Medicine, 33, 697-703. https://doi.org/10.7863/ultra.33.4.697

[14] Chauhan, S., Diamond, M.P. and Johns, D.A. (2004) A Case of Molar Ectopic Pregnancy. Fertility and Sterility, 81, 1140-1141.

https://doi.org/10.1016/j.fertnstert.2003.11.022

[15] Fowler, D.J., Lindsay, I. and Seckl, M.J. (2006) Routine Pre-Evacuation Ultrasound Diagnosis of Hydatidiform Mole: Experience of More than 1000 Cases from a Regional Referral Center. Ultrasound in Obstetrics \& Gynecology, 27, 56-60. https://doi.org/10.1002/uog.2592

[16] Husain, N., Mishra, S. and Pradeep, Y. (2008) Ectopic Molar Pregnancy Mimicking Choriocarcinoma. The Internet Journal of Gynecology and Obstetrics, 12, 10-11.

[17] Borah, T., Raphael, V., Panda, S. and Saharia, P. (2010) Ectopic Molar Pregnancy: A Rare Entity. Journal of Reproduction \& Infertility, 11, 201-203.

[18] Hoffmann, S., Abele, H. and Bachmann, C. (2016) Spontaneous Bilateral Tubal Ectopic Pregnancy: Incidental Finding During Laparoscopy-Brief Report and Review of Literature. Geburtshilfe Und Frauenheilkunde, 76, 413-416. https://doi.org/10.1055/s-0041-110394

[19] Beena, D., Teerthanath, S., Jose, V. and Shetty, J. (2016) Molar Pregnancy Presents as Tubal Ectopic Pregnancy: A Rare Case Report. Journal of Clinical and Diagnostic Research, 10, ED10-ED11. https://doi.org/10.7860/JCDR/2016/14875.7089

[20] Richards, A., Black, K. and Pather, S. (2013) Persistent Molar Pregnancy in an Ec- 
topic Treated with Laparoscopic Surgery: A Case Report. International Journal of Case Reports and Images, 4, 220-223.

[21] Mol, F., Van Mello, N.M. and Strandell, A. (2014) European Surgery in Ectopic Pregnancy (ESEP) Study Group. Salpingotomy versus Salpingectomy in Women with Tubal Pregnancy (ESEP Study): An Open-Label, Multicentre, Randomised Controlled Trial. The Lancet, 383, 1483-1489.

https://doi.org/10.1016/S0140-6736(14)60123-9 